
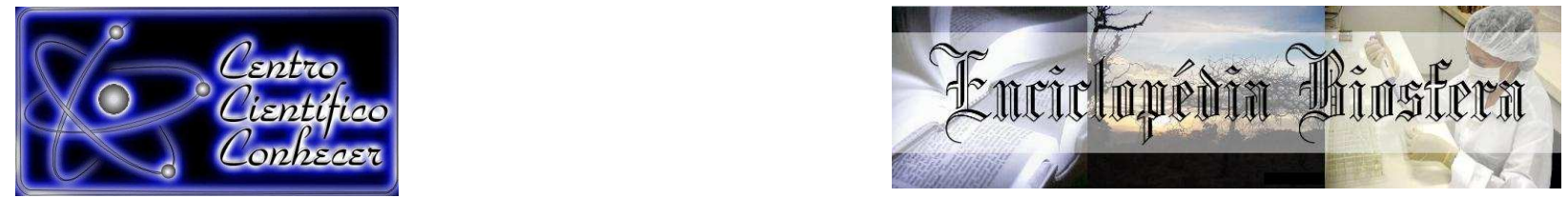

\title{
MANEJO DA ADUBAÇÃO NPK NA FORMAÇÃO DO AÇAIZEIRO EM LATOSSOLO AMARELO DO NORDESTE PARAENSE
}

\author{
Carlos Alberto Costa Veloso ${ }^{1}$, Arystides Resende Silva $^{2}$, Agust Sales $^{3}$ \\ ${ }^{1}$ Pesquisador Embrapa Amazônia Oriental, Laboratório de Solos, Belém, Brasil - \\ Pará (carlos.veloso@embrapa.br) \\ ${ }^{2}$ Pesquisador Embrapa Amazônia Oriental, Laboratório de Solos, Belém, Brasil - \\ Pará \\ ${ }^{3}$ Graduando em Engenharia Florestal da Universidade do Estado do Pará, \\ Paragominas - Brasil
} Recebido em: 08/09/2015 - Aprovado em: 14/11/2015 - Publicado em: 01/12/2015
DOI: http://dx.doi.org/10.18677/Enciclopedia_Biosfera_2015_190

\begin{abstract}
RESUMO
O açaizeiro possui grande importância no contexto agrícola paraense, porém dispõese de poucos resultados de pesquisa que proporcionem sustentação ao cultivo racional. $O$ objetivo deste trabalho foi determinar os níveis de nitrogênio, fósforo e potássio adequados para a fase de formação do açaizeiro em Latossolo Amarelo. 0 delineamento experimental utilizado foi o de blocos incompletos, no esquema fatorial fracionado $(4 \times 4 \times 4)^{1 / 2}$, com três repetições. Os tratamentos constituíram-se por um cultivo de açaí (Euterpe oleracea Mart.) sob aplicação de quatro doses de nitrogênio $(\mathrm{N})$, quatro de fósforo $\left(\mathrm{P}_{2} \mathrm{O}_{5}\right)$ e quatro de potássio $\left(\mathrm{K}_{2} \mathrm{O}\right)$ no primeiro e segundo ano de plantio, onde determinou-se a circunferência do coleto $(\mathrm{cm})$. Como base nos resultados, determinou-se as doses mais adequadas. Para o primeiro ano dos açaizeiros, 50 g.planta ${ }^{-1}$ de N, 55 g.planta ${ }^{-1}$ de $\mathrm{P}_{2} \mathrm{O}_{5}$ e 124 g.planta ${ }^{-1}$ de $\mathrm{K}_{2} \mathrm{O}$ juntamente com 30 g. planta $^{-1} \mathrm{MgO}$ e 10 g.planta $^{-1}$ de FTE - BR 12. Para o segundo ano, as doses mais adequadas foram $117 \mathrm{~g}$ de $\mathrm{N}, 158 \mathrm{~g}$ de $\mathrm{P}_{2} \mathrm{O}_{5}$ e 170 g.planta ${ }^{-1}$ de $\mathrm{K}_{2} \mathrm{O}$, juntamente com $30 \mathrm{~g}$ de $\mathrm{MgO}$ e $10 \mathrm{~g}$ de FTE. Confirmou-se a importância do NPK para o cultivo do açaizeiro. Entretanto, a frequência e quantidade de aplicação de NPK deve ser estudada respeitando as peculiaridades locais.
\end{abstract}

PALAVRAS-CHAVE: Adubação, Euterpe oleracea Mart., macronutriente.

\section{NPK FERTILIZER MANAGEMENT OF THE AÇAI PALM FORMATION IN NORTHEAST LATOSOL YELLOW PARAENSE}

\begin{abstract}
The açaí has great importance in Pará agricultural context, but it is had few search results that provide support to the rational cultivation. The objective of this study was to determine the levels of nitrogen, phosphorus and potassium suitable for açaizeiro the formation phase in Oxisoil Yellow. The experimental design was incomplete blocks, in fractional factorial $(4 \times 4 \times 4) \frac{1}{2}$, with three replications. The treatments were constituted by a açaí cultivation (Euterpe oleracea Mart.) Under application of four levels of nitrogen $(\mathrm{N})$, four of phosphorus $\left(\mathrm{P}_{2} \mathrm{O}_{5}\right)$ and four potassium $\left(\mathrm{K}_{2} \mathrm{O}\right)$ in the first and second year after planting, which determined themselves to collect circumference $(\mathrm{cm})$. As to the results, it was determined the most appropriate doses.
\end{abstract}


For the first year of açaizeiros 50 g.planta ${ }^{-1}$ of $\mathrm{N}, \mathrm{P}_{2} \mathrm{O}_{5}$ g.planta ${ }^{-1} 55$ and 124 g.planta ${ }^{1}$ one together with $30 \mathrm{~K}_{2} \mathrm{O}$ g.planta ${ }^{-1} \mathrm{MgO}$ and 10 g.planta ${ }^{-1} \mathrm{FTE}-\mathrm{BR} 12$. In the second year, the most appropriate doses were $117 \mathrm{~g}$ of $\mathrm{N}, \mathrm{P}_{2} \mathrm{O}_{5}$ and $170158 \mathrm{~g}$ g.planta ${ }^{-1} \mathrm{~K}_{2} \mathrm{O}, \mathrm{MgO}$ together with $30 \mathrm{~g}$ and $10 \mathrm{~g} \mathrm{FTE}$. He confirmed the importance of NPK açaizeiro for cultivation. However, the frequency and amount of NPK application should be studied respecting local peculiarities.

KEYWORDS: Fertilization, Euterpe oleracea Mart., macronutrient.

\section{INTRODUÇ̃̃O}

O açaizeiro (Euterpe oleracea Mart.) é uma espécie frutífera e produtora de palmito, nativa da Amazônia (OLIVEIRA et al., 2007). A produção nacional de açaí atinge aproximadamente 203 mil toneladas de frutos ao ano, sendo o Estado do Pará responsável por 55\% do total (IBGE, 2013), dados que comprovam a importância dessa fruteira para o Pará.

Atualmente, a demanda e o potencial de mercado do açaí cresceram de maneira significativa, deixando de possuir uma dimensão regional para ganhar importância nacional e mais recentemente internacional (SANTANA et al., 2008; Conab, 2014). O crescimento do mercado do açaizeiro está associado aos benefícios à saúde, que a ciência vem atribuindo à ingestão desse alimento que apresenta baixo nível de calorias, altas concentrações de vitaminas, fibras, sais minerais e à identificação de compostos com propriedades antioxidantes, por exemplo, carotenoides e flavonóides (KANG et al.; 2011 RUFINO et al.; 2011).

O aumento da área plantada com açaí na Amazônia, em função da demanda atual, promove seu cultivo racional. À medida que os cultivos vão se intensificando, o empobrecimento químico dos solos vai se tornando mais expressivo devido à extração dos nutrientes pelas plantas, exportação pelas colheitas e pelas perdas no solo via lixiviação, erosão e fixação reduzindo a disponibilidade desses nutrientes para as plantas (SANTANA et al., 2008).

Além disso, a grande maioria do cultivo do açaizeiro paraense está implantada em solos de baixa fertilidade natural e com alto teor de alumínio trocável, exigindo o emprego de corretivos e fertilizantes para uma produtividade sustentada (GONÇALVES \& MEURER, 2010).

O baixo consumo de fertilizantes e corretivos de acidez ainda é uma realidade na região amazônica (CUNHA et al., 2014), apesar desta prática representar uma forma de melhorar a fertilidade destes solos, de modo a proporcionar produtividades mais rentáveis aos agricultores. Destaca-se a aplicação de nutrientes em quantidades adequadas e em formas que possam ser assimiladas pelas plantas (WASTOWSKI et al., 2010). Desse modo, o objetivo deste trabalho foi determinar os níveis de nitrogênio, fósforo e potássio adequados para a fase de formação do açaizeiro em Latossolo Amarelo do Nordeste Paraense.

\section{MATERIAL E MÉTODOS}

O trabalho foi realizado em área de produtor no município de Inhangapi, Pará ( $1^{\circ} 20^{\prime} 54^{\prime \prime} \mathrm{S}$ e $47^{\circ} 54^{\prime} 38^{\prime \prime} \mathrm{W}$ a uma altitude de 38 metros). O solo foi classificado como Latossolo Amarelo textura média (EMBRAPA, 2006), cujos resultados da análise química de solo da área experimental na profundidade de $0-20 \mathrm{~cm}$ foram: $\mathrm{pH}$ (água) 4,4; P 2 mg.dm ${ }^{-3} ; \mathrm{K} 14 \mathrm{mg} \cdot \mathrm{dm}^{-3}$; $\mathrm{Ca}+\mathrm{Mg}$ 0,9 cmolc. $\mathrm{dm}^{-3}$; Al 0,8 cmolc. $\mathrm{dm}^{-3}$;

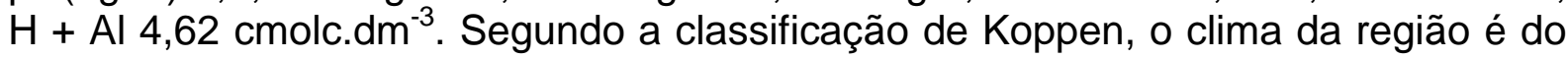
tipo Af. A precipitação apresenta média anual de $2383 \mathrm{~mm}$. A temperatura média anual varia entre $21,6^{\circ} \mathrm{C}$ e $32,5^{\circ} \mathrm{C}$ e a umidade relativa do ar média anual é de $85 \%$. 
O delineamento experimental utilizado foi o de blocos incompletos, no esquema fatorial fracionado $(4 \times 4 \times 4)^{1 / 2}$, com três repetições. Os tratamentos constituíram-se por um cultivo de açaí (Euterpe oleracea Mart.) sob aplicação de quatro doses de nitrogênio $(\mathrm{N})$, quatro de fósforo $\left(\mathrm{P}_{2} \mathrm{O}_{5}\right)$ e quatro de potássio $\left(\mathrm{K}_{2} \mathrm{O}\right)$ no primeiro e segundo ano de plantio.

Para o experimento utilizou-se um plantio de 288 plantas no espaçamento $6 \mathrm{x}$ $4 \mathrm{~m}$. No primeiro ano foram testadas quatro doses de $\mathrm{N}\left(0,30,60\right.$ e 90 g.planta $\left.{ }^{-1}\right)$, de $\mathrm{P}_{2} \mathrm{O}_{5}\left(0,40,80\right.$ e 120 g.planta $\left.{ }^{-1}\right)$ e de $\mathrm{K}_{2} \mathrm{O}\left(0,50,100\right.$ e 150 g.planta $\left.{ }^{-1}\right)$. Em função dos resultados obtidos no primeiro ano, foram definidas as doses para o segundo ano, sendo, $\mathrm{N}\left(0,60,120\right.$ e 180 g.planta $\left.^{-1}\right), \mathrm{P}_{2} \mathrm{O}_{5}(0,50,100$ e 150 g.planta $\left.{ }^{1}\right)$ e $4 \mathrm{~K}_{2} \mathrm{O}\left(0,100,200\right.$ e 300 g.planta $\left.{ }^{-1}\right)$.

Com exceção da testemunha, todos os tratamentos receberam lastro uniforme de sulfato de magnésio de 30 g.planta $^{-1}$ e de micronutrientes de 10 g.planta ${ }^{-1}$ de FTE BR 13. O sulfato de magnésio foi aplicado em duas porções iguais, na mesma ocasião da adubação de $\mathrm{N}$ e $\mathrm{K}_{2} \mathrm{O}$. O FTE e o fósforo fornecido como fosfato natural (Arad) foram aplicados em dose total. O nitrogênio fornecido como uréia e o potássio como cloreto, em duas parcelas iguais.

Utilizou-se parcelas experimentais formadas por 8 plantas em cada linha, sendo as 6 plantas centrais consideradas úteis, onde determinou-se a circunferência do coleto $(\mathrm{cm})$. Os resultados foram avaliados estatisticamente, no programa estatístico SISVAR (FERREIRA, 2011) através de análise de variância (teste F), para todas as variáveis. Curvas de regressão foram ajustadas para avaliação das respostas a aplicação de $\mathrm{N}, \mathrm{P}, \mathrm{K}$ em função das variáveis.

\section{RESULTADOS E DISCUSSÃO}

A aplicação das doses de nitrogênio tendo como fonte a ureia, referente ao primeiro ano, promoveu redução progressiva nos valores de circunferência do coleto (CDC) à medida que aumentou-se a dosagem (Figura 1). A dose máxima estimada de nitrogênio foi de 50 g.planta ${ }^{-1}$. A dose de 50 g.planta ${ }^{-1}$ é compatível com a recomendação de VIÉGAS \& BOTELHO (2007), para açaizeiros com um ano de idade que é de 45 g.planta ${ }^{-1}$ de $\mathrm{N}$.
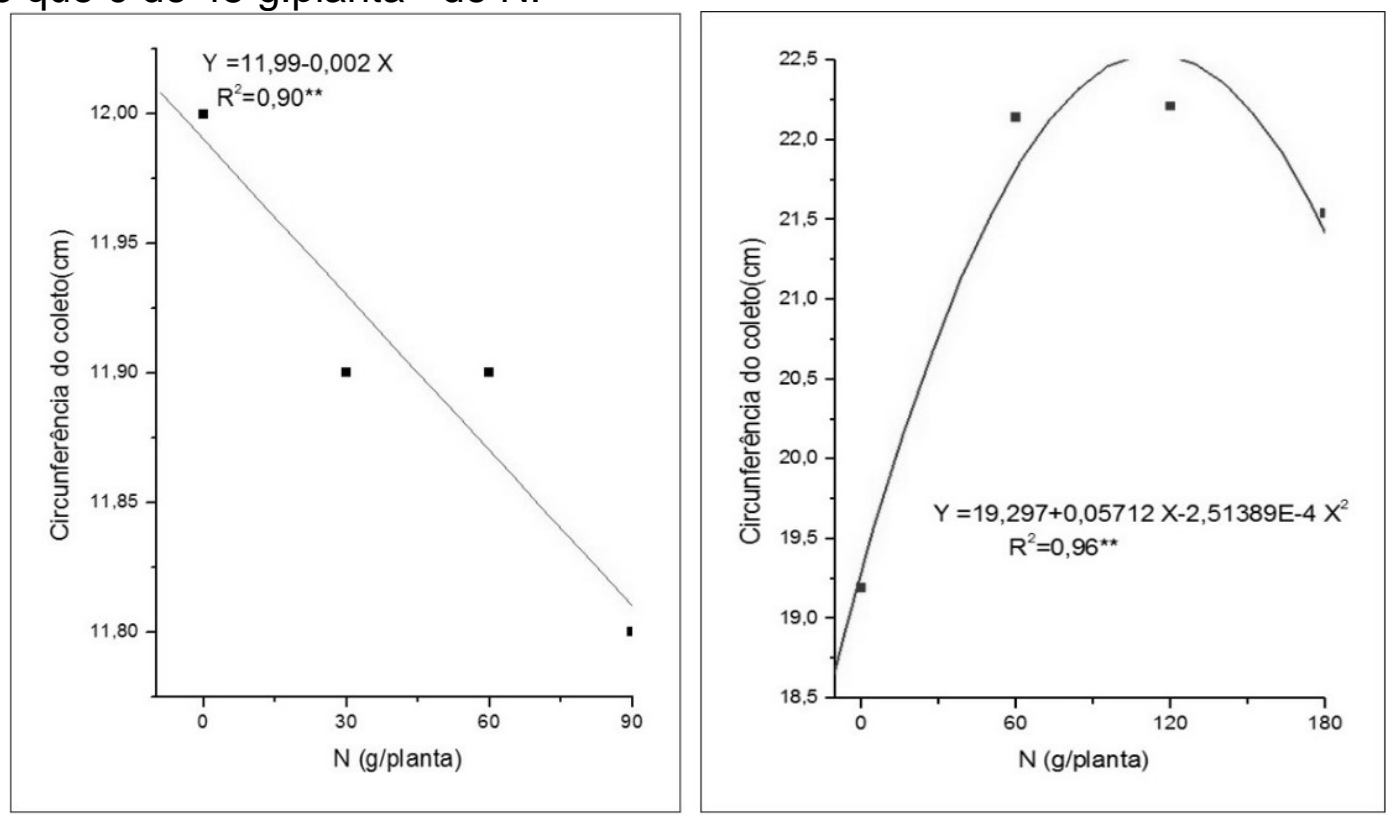

FIGURA 1. Efeito das doses de nitrogênio sobre a circunferência do coleto (CDC) do açaizeiro com um e dois anos de idade. 
A aplicação de fósforo promoveu aumento para a variável CDC até 56 g.planta ${ }^{-1}$, apresentando redução de CDC as dosagens superiores (Figura 2). A dose máxima estimada de fósforo foi de 55,3 g.planta ${ }^{-1}$ de $\mathrm{P}_{2} \mathrm{O}_{5}$ que correspondeu a 12,3 $\mathrm{cm}$ de CDC (Figura 2). Essa dose (55.3 g.planta ${ }^{-1}$ ) de $\mathrm{P}_{2} \mathrm{O}_{5}$ é também compatível, apenas inferior em 4,7 g.planta-1 ${ }^{-1}$ à recomendada por VIÉGAS \& BOTELHO (2007), para o açaizeiro no primeiro ano em solo com 0 a $10 \mathrm{mg} \mathrm{dm}^{-3}$ de fósforo.

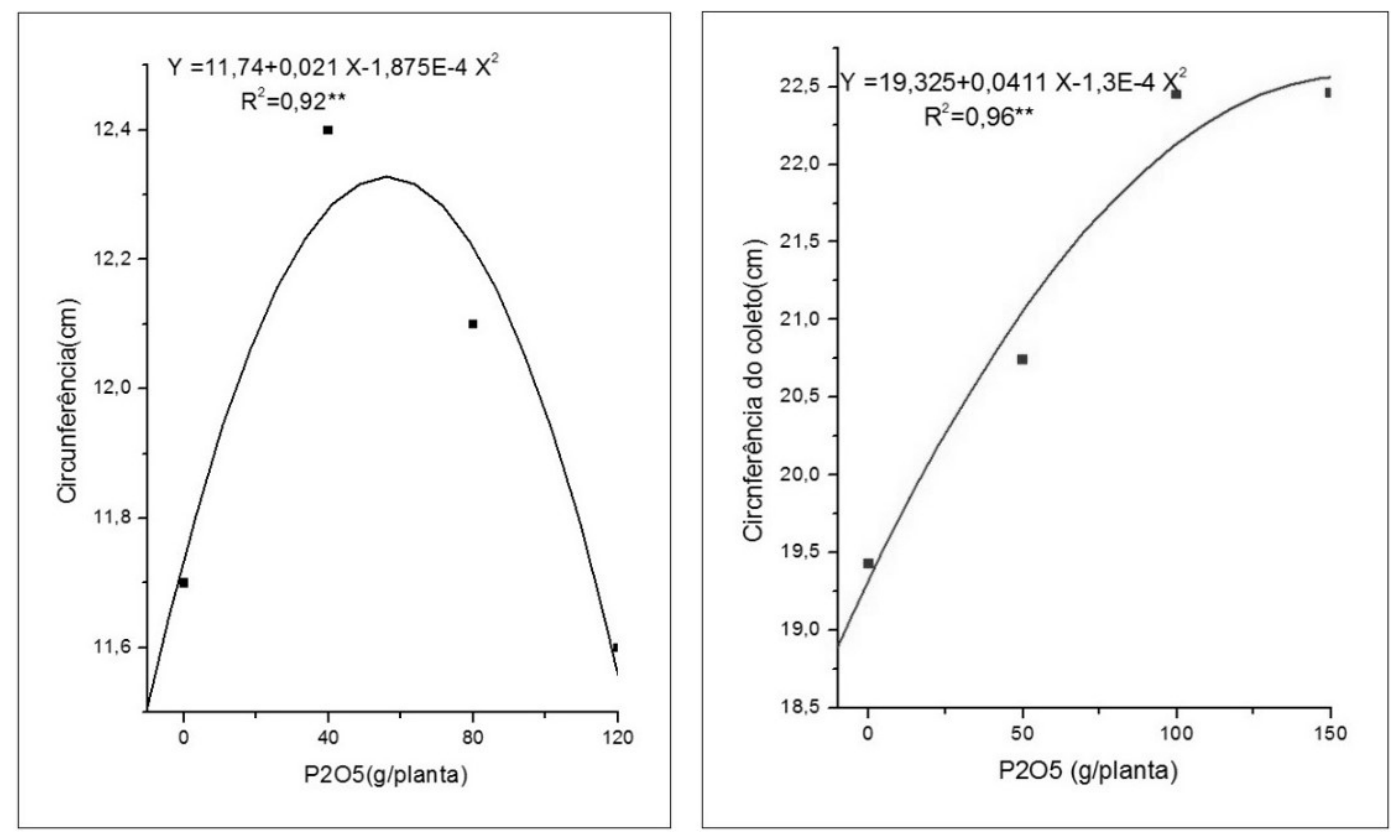

FIGURA 2. Efeito das doses de fósforo sobre a circunferência do coleto (CDC) do açaizeiro com um e dois anos de idade.

A aplicação de potássio, tendo como fonte o cloreto de potássio, indicou aumentou gradual de CDC com o aumento da dosagem (Figura 3). A dose de 124,4 g.planta ${ }^{-1}$ de $\mathrm{K}_{2} \mathrm{O}$ para açaizeiros com um ano de idade é superior à recomendada por VIÉGAS \& BOTELHO (2007), que é de 80 g.planta $^{-1}$ de $\mathrm{K}_{2} \mathrm{O}$ com teor no solo de 0 a $40 \mathrm{mg} \cdot \mathrm{dm}^{-3}$ de $\mathrm{K}_{2} \mathrm{O}$.
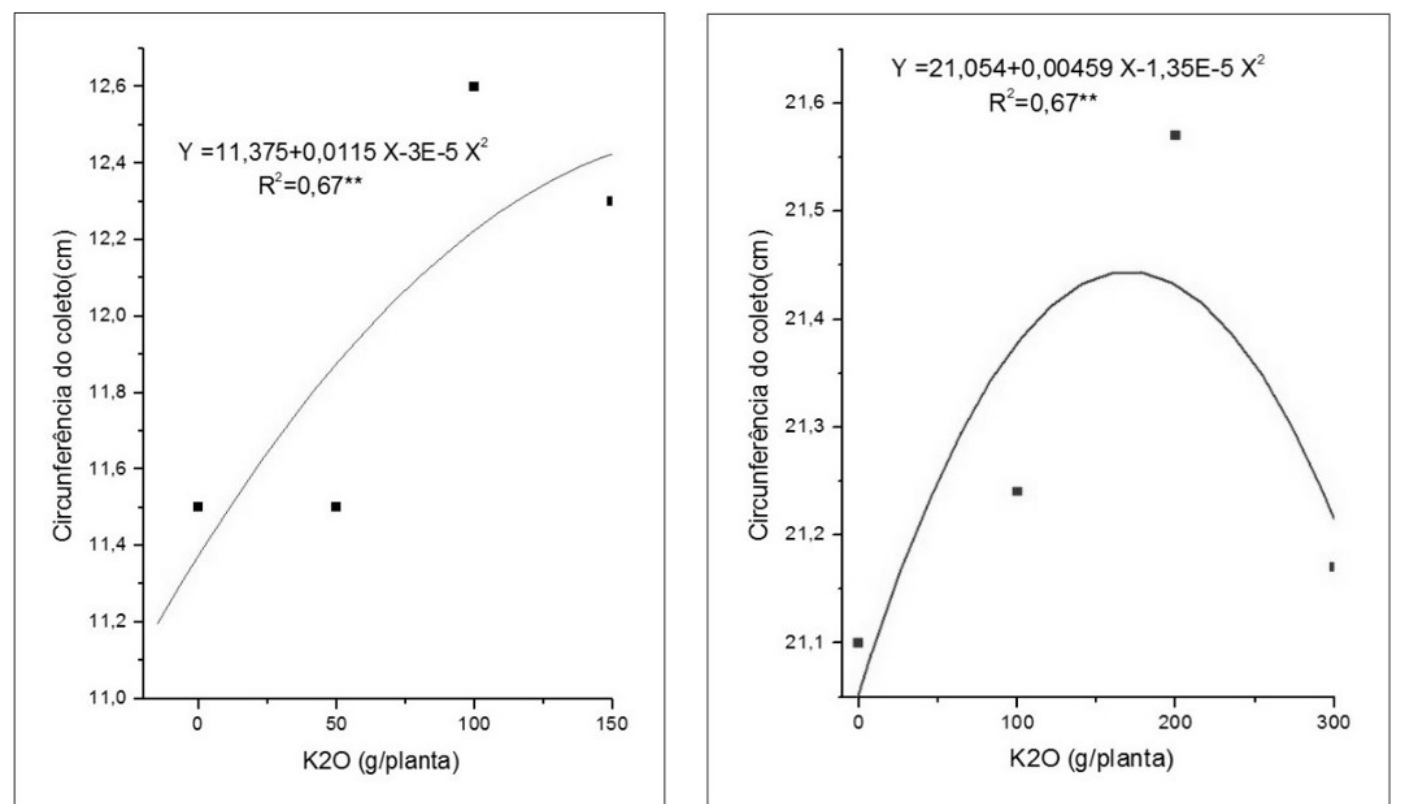

FIGURA 3. Efeito das doses de potássio sobre a circunferência do coleto (CDC) do açaizeiro com um e dois anos de idade. 
Resultados semelhantes aos observados por VIEIRA et al. (2013), ao avaliarem 0 crescimento em altura e diâmetro de mudas de Schizolobium amazonicum submetidas a diferentes níveis de adubações com NPK, atestaram influência de doses de NPK no crescimento das mudas. Diferentemente dos resultados obtidos por TUCCI et al. (2011), no estudo em que avaliaram mudas de Swietenia macrophylla em resposta à NPK, onde a adubação com nitrogênio e fósforo não promoveu melhora no desenvolvimento das mudas.

PEZZUTTI et al. (1999), no estudo em que avaliaram o crescimento de mudas de Eucalyptus globulus em resposta a fertilização NPK, também observaram influência do NPK no crescimento de mudas, indicando que o diâmetro do coleto respondeu positivamente à adubação NPK.

A adubação nitrogenada no segundo ano promoveu aumento de CDC, gerando redução de CDC a partir da dosagem de 120 g.planta $^{-1}$ (Figura 1). A dose máxima estimada de nitrogênio para a CDC foi de 113,6 g.planta ${ }^{-1}$ que correspondeu a $22,54 \mathrm{~cm}$. Essa dose é superior à recomendada por VIÉGAS \& BOTELHO (2007), para açaizeiros com dois anos de idade, que é de 80 g.planta ${ }^{-1}$ de Nitrogênio. Estes resultados demonstram o efeito do nitrogênio na formação do açaizeiro, visto que está intimamente ligado nos processos fisiológicos como a fotossíntese e a respiração (MENGEL \& KIRKBY, 1987).

Os efeitos da adubação fosfatada no segundo ano, a exemplo do nitrogênio também promoveram incremento de CDC com o aumento da dosagem (Figura 2). A dose máxima estimada de fósforo para a CDC foi de 158 g.planta ${ }^{-1}$ que correspondeu a $22,57 \mathrm{~cm}$. O incremento de CDC em função da adubação fosfatada é devido o fósforo ser necessário para a síntese do ATP e de numerosos outros compostos fosforilados (EPSTEIN, 1975).

Os efeitos da adubação potássica no segundo ano promoveu aumento de CDC, apresentando redução de CDC a partir da dose de 200 g.planta $^{-1}$ (Figura 3). A dose máxima estimada de potássio para a CDC foi de 170 g.planta ${ }^{-1}$ de $\mathrm{K}_{2} \mathrm{O}$. Essas doses obtidas de potássio são superiores a recomendada por VIÉGAS E BOTELHO (2007), para açaizeiros com dois anos de idade com teor no solo de 0 a $40 \mathrm{mg} \cdot \mathrm{dm}^{-3}$ de $\mathrm{K}$ que é de 100 g.planta ${ }^{-1}$ de $\mathrm{K}_{2} \mathrm{O}$.

CRUZ et al. (2010), observaram efeitos significativos da aplicação adubação potássica em mudas de fedegoso (Senna macranthera) indicando ser essa espécie florestal mais exigente nutricionalmente em potássio que a espécie desse estudo.

Resultados que concordam com GOMES \& PAIVA (2006), onde observaram efeito positivo com níveis crescentes de potássio em mudas florestais, visto que o potássio atua na ativação de várias enzimas e regulação do potencial osmótico das células (EVANS \& SORGER, 1966). CAIONE et al. (2012), ao avaliarem o efeito da aplicação de nitrogênio, fósforo e potássio no crescimento de mudas de pinhocuiabano, verificaram que o tratamento completo, com NPK, possibilitou maior diâmetro do coleto das mudas, apresentando resultados superiores aos demais tratamentos.

LOCATELLI et al. (2007), no estudo onde avaliaram deficiências nutricionais em mudas de Schizolobium parahyba var. amazonicum, verificaram que as deficiências de NPK levaram ao maior decréscimo no desenvolvimento das mudas. Já os autores SOUZA et al. (2006), relatam que vários pesquisadores estimam a sobrevivência de mudas de diferentes espécies florestais no período pós-plantio de acordo com o diâmetro do coleto, sendo o mesmo considerado um importante parâmetro. Entretanto, a literatura não apresenta parâmetros para avaliação de um 
bom diâmetro para a espécie em estudo e, as dosagens recomendadas no presente trabalho são superiores às recomendadas por VIÉGAS \& BOTELHO (2007).

Os dois primeiros anos de cultivo do açaizeiro indicaram que o NPK é importante para o açaizeiro, haja vista a ocorrência de menores valores de CDC nos tratamentos sem aplicação desses nutrientes. Como base nos resultados, determinaram-se as doses mais adequadas. Para o primeiro ano dos açaizeiros, 50 g.planta ${ }^{-1}$ de $\mathrm{N}, 55$ g.planta ${ }^{-1}$ de $\mathrm{P}_{2} \mathrm{O}_{5}$ e 124 g.planta ${ }^{-1}$ de $\mathrm{K}_{2} \mathrm{O}$ juntamente com 30 g.planta ${ }^{-1} \mathrm{MgO}$ e 10 g.planta ${ }^{-1}$ de FTE - BR 12. E para o segundo ano, as doses mais adequadas foram $117 \mathrm{~g}$ de $\mathrm{N}, 158 \mathrm{~g}$ de $\mathrm{P}_{2} \mathrm{O}_{5}$ e 170 g.planta ${ }^{-1}$ de $\mathrm{K}_{2} \mathrm{O}$, juntamente com $30 \mathrm{~g}$ de $\mathrm{MgO}$ e $10 \mathrm{~g}$ de FTE.

\section{CONCLUSÃO}

Confirmou-se a importância do NPK para o cultivo do açaizeiro. Contudo, a frequência e quantidade de aplicação de NPK devem ser estudadas de acordo com as particularidades locais, pois acredita-se que elevadas aplicações anuais desses elementos podem contribuir negativamente na formação do açaizeiro.

\section{REFERÊNCIAS}

CAIONE, G.; LANGE, A.; SCHONINGER, E. L. Crescimento de mudas de Schizolobium amazonicum (Huber ex Ducke) em substrato fertilizado com nitrogênio, fósforo e potássio. Scientia Forestalis. Piracicaba, v. 40, n. 94, p. 213-221, 2012.

COMPANHIA NACIONAL DE ABASTECIMENTO (CONAB). 2014. Conjuntura Mensal - Açaí (fruto). Disponível em: <http://www.conab.gov.br/OlalaCMS/up loads/arquivos/14 $08 \quad 05 \quad 1107 \quad 18$ acai julho 2014.pdf> Acesso em: 16 abr. 2015.

CRUZ, C. A. F.; PAIVA, H. N.; NEVES, J. C. L.; CUNHA, A. C. M. C. M. Resposta de mudas de Senna macranthera (Dc. Ex Collad.) H.S. Irwin \& Barnaby (Fedegoso) cultivadas em Latossolo Vermelho-amarelo distrófico a macronutrientes. Revista Árvore, Viçosa, v. 34, n. 1, p. 13-24, 2010.

CUNHA, J. F.; FRANCISCO, E. A. B; CASARIN, V.; PROCHNOW, L. I. Balanço de nutrientes na agricultura brasileira - 2009 a 2012. Informações agronômicas. n145, 28 p. 2014.

EMPRESA BRASILEIRA DE PESQUISA AGROPECUÁRIA (EMBRAPA). Centro Nacional de Pesquisa de Solos. Sistema brasileiro de classificação de solos. 2. ed. Rio de Janeiro: Embrapa Solos. 2006.

EPSTEIN, E. Nutrição mineral de plantas: princípios e perspectivas. São Paulo: USP, 1975. 344p.

EVANS, H. J.; SORGER, G. J. Role of mineral elements with emphasis on the univalent 318 cations. Annual Review of Plant Physiology, Palo Alto, v.17, p.4776. 1966.

FERREIRA, D. F. Sisvar: a computer statistical analysis system. Ciência e Agrotecnologia (UFLA), v. 35, n.6, p. 1039-1042, 2011. 
GOMES, J. M.; PAIVA, H. N. Viveiros Florestais: propagação sexuada. Viçosa: UFV, 2006.

GONÇALVES, G. K.; MEURER, E. J. Alterações nas concentrações de fósforo em solos cultivados com arroz irrigado no Rio Grande do Sul. Revista Brasileira de Ciência do Solo, Porto Alegre, v. 34, p. 465-471. 2010.

INSTITUTO BRASILEIRO DE GEOGRAFIA E ESTATÍSTICA (IBGE). 2013. Produção da extração vegetal e da silvicultura. Rio de Janeiro, v. 28, p. 1-69. Disponível em: <http://www.ibge.gov.br/home/estatistica/ economia/pevs/2013/ default pdf.shtm>. Acesso em: 08 abr. 2014.

KANG, J.; XIE, C.; LI, Z.; NAGARAJAN, S.; SCHAUSS, A. G.; WU, T.; WU, X. Flavonoids from açaí (Euterpe oleracea Mart.) pulp and their antioxidante and antiinflammatory activities. Food Chemistry, Barking, v. 120, p.152-157. 2011.

LOCATELLI, M.; MELO, A. S.; LIMA, L. M. L.; VIEIRA, A. H. Deficiências nutricionais em mudas de Schizolobium parahyba var. amazonicum. Revista Brasileira de Biociências, Porto Alegre, v. 5, n. 2, p. 648-650, 2007.

MENGEL, K.; KIRKBY, E. Principles of plan nutrition. 4ed. Oxford: International Potash Institute, 1987. 687p.

OLIVEIRA, M. S. P.; FARIAS NETO, J. T.; PENA, R. S. Açaí: técnicas de cultivo e processamento. Fortaleza: Instituto Frutal, p.104. 2007.

PEZZUTTI, R. V.; SCHUMACHER, M. V.; HOPPE, J. M. Crescimento de mudas de Eucalyptus globulus em resposta a fertilização NPK. Ciência Florestal, Santa Maria, v. 9, n. 2, p. 117-125, 1999.

RUFINO, M. S. M. PÉREZ-JIMÉNEZ, J.; ARRANZ, S.; ALVES, R. E.; BRITO, E. S.; OLIVEIRA, M. S. P.; SAURA-CALIXTO, F. Açaí (Euterpe oleraceae) "BRS Pará": A tropical fruit source of antioxidante dietary fiber and high antioxidant capacity oil. Food Research International, Barking, v. 44, n. 7, p. 2100-2106. 2011.

SANTANA, A. C.; CARVALHO, D. F.; MENDES, F. A. T. Análise sistêmica da fruticultura paraense: organização, mercado e competitividade empresarial. Belém: Banco da Amazônia, 255 p. 2008.

SOUZA, C. A. M.; OLIVEIRA, R. B.; MARTINS FILHO, S.; SOUZA, J. L. S. Crescimento em campo de espécies florestais em diferentes condições de adubações. Ciência Florestal, Santa Maria, v. 16, n. 3, p. 243-249, 2006.

TUCCI, C. A. F.; SANTOS, J. Z. L.; SILVA JUNIOR, C. H.; SOUZA, P. A.; BATISTA, I. M. P.; VENTURIN, N. Desenvolvimento de mudas de Swietenia macrophylla em resposta a nitrogênio, fósforo e potássio. Floresta, Curitiba, v. 41, n .3, p.471-490, 2011. 
VIÉGAS, L. J. M.; BOTELHO, S. M. Açaizeiro. In: Recomendações de adubação e calagem para o estado do Pará. Ed. Técnicos, CRAVO, M. da Silva; VIÉGAs, I DE J.M.; BRASIL, E. C. Belém, PA: Embrapa Amazônia Oriental, 262p. 2007.

VIEIRA, C. R.; WEBER, O. L. S.; SCARAMUZZA, J. F. Influência da adubação NPK no crescimento em altura e diâmetro de mudas de Schizolobium amazonicum. In: IV CONGRESSO BRASILEIRO DE GESTÃO AMBIENTAL, 1, 2013, Salvador, Anais... IBEAS. Salvador: 2013.

WASTOWSKI, A. D.; ROSA, G. M.; CHERUBIN, M. R.; RIGON, J. P. G. Caracterização dos níveis de elementos químicos em solo, submetido a diferentes sistemas de uso e manejo, utilizando espectrometria de fluorescência de raios-x por energia dispersiva (edxrf). Química Nova, v. 33, n. 7, p. 1449-1452. 2010. 